The Journal of $\mathbf{N}_{\text {onlinear }} \mathbf{S}_{\text {ciences and }}$ Applications http://www.tjnsa.com

\title{
FUZZY MINIMAL SEPARATION AXIOMS
}

\author{
M. ALIMOHAMMADY ${ }^{1}$, E. EKICI ${ }^{2, *}$, S. JAFARI $^{3}$, M. ROOHI $^{1}$
}

ABstract. In this paper, we deal with some separation axioms in the context of fuzzy minimal structures.

\section{Introduction}

Zadeh introduced the concept of a fuzzy set in [14]. Subsequently, many attempts have been made to extend many science notions to the fuzzy setting, for example [8, 10, 11]. Fuzzy minimal structure and fuzzy minimal space introduced and investigated in [1-7]. For easy understanding of the material incorporated in this paper, we recall some basic definitions and results. For details on the following notions we refer to [1-14] and the references cited therein.

A fuzzy set in(on) a universe set $X$ is a function with domain $X$ and values in $I=[0,1]$. The class of all fuzzy sets on $X$ will be denoted by $I^{X}$ and symbols $A, B, \ldots$ is used for fuzzy sets on $X .01_{X}$ is called empty fuzzy set where $1_{X}$ is the characteristic function on $X$. A family $\mathcal{M}$ of fuzzy sets in $X$ is said to be a fuzzy minimal structure in Chang's sense on $X$ if $\left\{01_{X}, 1_{X}\right\} \subseteq \mathcal{M}$. In this case $(X, \mathcal{M})$ is called a fuzzy minimal space [2]. A fuzzy set $A \in I^{X}$ is said to be fuzzy $m$-open if $A \in \mathcal{M} . B \in I_{X}$ is called a fuzzy $m$-closed set if $B^{c} \in \mathcal{M}$. Let

$$
\begin{aligned}
m-\operatorname{Int}(A) & =\bigvee\{U: U \leq A, U \in \mathcal{M}\} \text { and } \\
m-C l(A) & =\bigwedge\left\{F: A \leq F, F^{c} \in \mathcal{M}\right\} .
\end{aligned}
$$

A fuzzy set in $X$ is called a fuzzy point if it takes the value 0 for all $y \in X$ except one, say, $x \in X$. If its value at $x$ is $\alpha(0<\alpha \leq 1)$, we denote this fuzzy point by $x_{\alpha}$, where the point $x$ is called its support [12, 13]. For any fuzzy point

Date: Revised: 03, May, 2010.

* Corresponding author

(c) 2010 N.A.G.

Key words and phrases. Fuzzy sets, fuzzy topology, fuzzy separation axiom. 
$x_{\alpha}$ and any fuzzy set $A, x_{\alpha} \in A$ if and only if $\alpha \leq A(x)$. A fuzzy point $x_{\alpha}$ is called quasi-coincident with a fuzzy set $B$, denoted by $x_{\alpha} q B$, if $\alpha+B(x)>1$. A fuzzy set $A$ is called quasi-coincident with a fuzzy set $B$, denoted by $A q B$, if there exists a $x \in X$ such that $A(x)+B(x)>1$ [12, 13]. When they are not quasi-coincident, it will denoted by $A \not q B$. Throughout in this paper we assume that all fuzzy minimal spaces are in the sense of Chang.

Proposition 1.1. [2] For any two fuzzy sets $A$ and $B$ in a fuzzy minimal space $(X, \mathcal{M})$

(1) $m-\operatorname{Int}(A) \leq A$ and $m-\operatorname{Int}(A)=A$ if $A$ is a fuzzy m-open set.

(2) $A \leq m-C l(A)$ and $A=m-C l(A)$ if $A$ is a fuzzy $m$-closed set.

(3) $m-\operatorname{Int}(A) \leq m-\operatorname{Int}(B)$ and $m-C l(A) \leq m-C l(B)$ if $A \leq B$.

(4) $(m-\operatorname{Int}(A)) \wedge(m-\operatorname{Int}(B)) \leq m-\operatorname{Int}(A \wedge B)$ and $(m-\operatorname{Int}(A)) \vee(m-$ $\operatorname{Int}(B)) \leq m-\operatorname{Int}(A \vee B)$.

(5) $(m-C l(A)) \vee(m-C l(B)) \leq m-C l(A \vee B)$ and $m-C l(A \wedge B) \leq$ $(m-C l(A)) \wedge(m-C l(B))$.

(6) $m-\operatorname{Int}(m-\operatorname{Int}(A))=m-\operatorname{Int}(A)$ and $m-C l(m-C l(B))=m-C l(B)$.

(7) $(m-C l(A))^{c}=m-\operatorname{Int}\left(A^{c}\right)$ and $(m-\operatorname{Int}(A))^{c}=m-C l\left(A^{c}\right)$.

Definition 1.2. [2] A fuzzy minimal space $(X, \mathcal{M})$ enjoys the property $U$ if arbitrary union of fuzzy $m$-open sets is fuzzy $m$-open.

Proposition 1.3. [1] For a fuzzy minimal structure $\mathcal{M}$ on a set $X$, the following statements are equivalent.

(1) $(X, \mathcal{M})$ has the property $U$.

(2) If $m-\operatorname{Int}(A)=A$, then $A \in \mathcal{M}$.

(3) If $m-C l(B)=B$, then $B^{c} \in \mathcal{M}$.

Fuzzy minimal continuous functions was introduced and studied in [3].

Definition 1.4. 3] Let $(X, \mathcal{M})$ and $(Y, \mathcal{N})$ be two fuzzy minimal spaces. We say that a fuzzy function $f:(X, \mathcal{M}) \rightarrow(Y, \mathcal{N})$ is fuzzy minimal continuous (briefly fuzzy $m$-continuous) if $f^{-1}(B) \in \mathcal{M}$, for any $B \in \mathcal{N}$.

Theorem 1.5. [3] Consider the following properties for a fuzzy function $f$ : $(X, \mathcal{M}) \rightarrow(Y, \mathcal{N})$ between two fuzzy minimal spaces.

(1) $f$ is a fuzzy m-continuous function.

(2) $f^{-1}(B)$ is a fuzzy $m$-closed set for each fuzzy $m$-closed set $B \in I^{Y}$.

(3) $m-C l\left(f^{-1}(B)\right) \leq f^{-1}(m-C l(B))$ for each $B \in I^{Y}$.

(4) $f(m-C l(A)) \leq m-C l(f(A))$ for any $A \in I^{X}$.

(5) $f^{-1}(m-\operatorname{Int}(B)) \leq m-\operatorname{Int}\left(f^{-1}(B)\right)$ for each $B \in I^{Y}$.

Then $(1) \Leftrightarrow(2) \Rightarrow(3) \Leftrightarrow(4) \Leftrightarrow(5)$. Moreover, if $(X, \mathcal{M})$ satisfies in the property $U$, then all of the above statements are equivalent. 


\section{Fuzzy minimal separation axioms}

Definition 2.1. A fuzzy set $N$ in a fuzzy minimal space $(X, \mathcal{M})$ is said to be a fuzzy minimal neighborhood of a fuzzy point $x_{\alpha}$ if there is a fuzzy $m$-open set $\mu$ in $X$ with $x_{\alpha} \in \mu$ and $\mu \leq N$.

Definition 2.2. Suppose $(X, \mathcal{M})$ is a fuzzy minimal space. A fuzzy set $N$ in $X$ is said to be a fuzzy minimal q-neighborhood of a fuzzy point $x_{\alpha}$ if there is a fuzzy $m$-open set $\mu$ in $X$ with $x_{\alpha} q \mu$ and $\mu \leq A$.

Definition 2.3. Suppose $(X, \mathcal{M})$ is a fuzzy minimal space. A fuzzy point $x_{\alpha}$ in $X$ is said to be fuzzy minimal cluster point of a fuzzy set $A$ if every fuzzy minimal $q$-neighborhood of $x_{\alpha}$ is $q$-coincident with $A$.

Theorem 2.4. Suppose $(X, \mathcal{M})$ is a fuzzy minimal space. A fuzzy point $x_{\alpha}$ is a fuzzy minimal cluster point of a fuzzy set $A$ if and only if $x_{\alpha} \in m-C l(A)$.

Proof. Suppose $x_{\alpha} \notin m-C l(A)$. Then, one can easily see that there exists $m$-closed set $F$ in $X$ with $A \leq F$ and $F(x)<\alpha$. Therefore, $x_{\alpha} q F^{c}$ and $A \not q F^{c}$; i.e., $x_{\alpha}$ is not a fuzzy minimal cluster point of $A$. Conversely, suppose $x_{\alpha}$ is not a fuzzy minimal cluster point of $A$. There exists a fuzzy minimal $q$-neighborhood $N$ of $x_{\alpha}$ for which $N \not q A$. Then there exists a fuzzy $m$-open set $\mu$ in $X$ with $x_{\alpha} q \mu$ and $\mu \leq N$. Therefore, $\mu \not q A$ which implies that $A \leq \mu^{c}$. Since $\mu^{c}$ is $m$-closed, so (1.2) implies that $m-C l(A) \leq \mu^{c}$. That $x_{\alpha} \notin m-C l(A)$ follows from the fact that $x_{\alpha} \notin \mu^{c}$.

Definition 2.5. A fuzzy minimal space $(X, \mathcal{M})$ is said to be

(1) fuzzy minimal $T_{0}$ if for every pair of distinct fuzzy points $x_{\alpha}$ and $x_{\beta}$, when $x \neq y$ either $x_{\alpha}$ has a fuzzy minimal neighborhood which is not $q$-coincident with $y_{\beta}$ or $y_{\beta}$ has a fuzzy minimal neighborhood which is not $q$ coincident with $x_{\alpha}$,

when $x=y$ and $\alpha<\beta$ (say), there is a fuzzy minimal $q$-neighborhood of $y_{\beta}$ which is not $q$-coincident with $x_{\alpha}$,

(2) fuzzy minimal $T_{1}$ if for every pair of distinct fuzzy points $x_{\alpha}$ and $x_{\beta}$, when $x \neq y$ there is a fuzzy minimal neighborhood $\mu$ of $x_{\alpha}$ and a fuzzy minimal neighborhood $\nu$ of $y_{\beta}$ with $\mu \not q y_{\beta}$ and $x_{\alpha} \not q \nu$,

when $x=y$ and $\alpha<\beta$ (say), $y_{\beta}$ has a fuzzy minimal $q$-neighborhood which is not $q$-coincident with $x_{\alpha}$,

(3) fuzzy minimal $T_{2}$ if for every pair of distinct fuzzy points $x_{\alpha}$ and $x_{\beta}$, when $x \neq y, x_{\alpha}$ and $y_{\beta}$ have fuzzy minimal $q$-neighborhoods which are not $q$-coincident,

when $x=y$ and $\alpha<\beta$ (say), $x_{\alpha}$ has a fuzzy minimal neighborhood $\mu$ and $y_{\beta}$ has a fuzzy minimal $q$-neighborhood $\nu$ in which $\mu \not q \nu$.

In short fuzzy $m-T_{i}(\mathrm{i}=0,1,2)$ spaces are used for fuzzy minimal $T_{i}$ spaces.

Theorem 2.6. Every fuzzy $m-T_{2}$ space is a fuzzy $m-T_{1}$ space and also every fuzzy $m-T_{1}$ space is a fuzzy $m-T_{0}$ space. 
Proof. Obvious.

Theorem 2.7. A fuzzy minimal space $(X, \mathcal{M})$ is fuzzy $m-T_{1}$ if every fuzzy point $x_{\alpha}$ is fuzzy $m$-closed in $X$.

Proof. Suppose $x_{\alpha}$ and $y_{\beta}$ are distinct fuzzy points in $X$, there are two cases

(i) $x \neq y$

(ii) $x=y$ and $\alpha<\beta$ (say).

Assume that $x \neq y$. By hypothesis $x_{\alpha}^{c}$ and $y_{\beta}^{c}$ are fuzzy $m$-open sets. It is easy to see that $x_{\alpha} \in y_{\beta}^{c}, y_{\beta} \in x_{\alpha}^{c}, x_{\alpha} \not q x_{\alpha}^{c}$ and $y_{\beta} \not q y_{\beta}^{c}$. In case that $x=y$ and $\alpha<\beta$, one can deduce that $x_{\alpha}^{c}$ is a fuzzy $m$-open set with $y_{\beta} q x_{\alpha}^{c}$ and $x_{\alpha} \not q x_{\alpha}^{c}$ which implies that $(X, \mathcal{M})$ is fuzzy $m-T_{1}$.

Theorem 2.8. Let $(X, \mathcal{M})$ be a fuzzy minimal space. Then $(X, \mathcal{M})$ is fuzzy minimal $T_{1}$ if for each $x \in X$ and each $\alpha \in[0,1]$ there exists a fuzzy minimal open set $\mu$ such that $\mu(x)=1-\alpha$ and $\mu(y)=1$ for $y \neq x$.

Proof. Let $x_{\alpha}$ be an arbitrary fuzzy point of $X$. We shall show that the fuzzy point $x_{\alpha}$ is fuzzy minimal closed. By hypothesis, there exists a fuzzy minimal open set $\mu$ such that $\mu(x)=1-\alpha$ and $\mu(y)=1$ for $y \neq x$. We have $\mu^{c}=x_{\alpha}$. Thus, the fuzzy point $x_{\alpha}$ is fuzzy minimal closed and hence by Theorem 2.7 the fuzzy minimal space $X$ is fuzzy minimal $T_{1}$.

Theorem 2.9. Suppose $i=0,1,2$. A fuzzy minimal space $(X, \mathcal{M})$ is fuzzy $m-T_{i}$ if and only if for any pair of distinct fuzzy points $x_{\alpha}$ and $y_{\beta}$ with distinct supports, there exists a fuzzy $m$-continuous mapping $f$ from $X$ into a fuzzy $m-T_{i}$ space $(Y, \mathcal{N})$ such that $f(x) \neq f(y)$.

Proof. We only prove the case that $i=2$ and others are similar. Suppose $(X, \mathcal{M})$ is fuzzy $m-T_{2}$ space. Let $(Y, \mathcal{N}):=(X, \mathcal{M})$ and $f:=i d_{X}$. Clearly, $(Y, \mathcal{N})$ and $f$ have the required properties. Conversely, suppose $x_{\alpha}$ and $y_{\beta}$ are distinct fuzzy points in $X$. There are two cases

(i) $x \neq y$

(ii) $x=y$ and $\alpha<\beta$ (say).

When $x \neq y$, by assumption there is fuzzy $m$-continuous mapping $f$ from $(X, \mathcal{M})$ into a fuzzy $m-T_{2}$ space $(Y, \mathcal{N})$ with $f(x) \neq f(y)$. Since $(Y, \mathcal{N})$ is fuzzy $m-T_{2}$ space and $(f(x))_{\alpha}$ and $(f(y))_{\beta}$ are distinct fuzzy points in $Y$, so there are fuzzy minimal neighborhoods $\mu$ and $\nu$ of $(f(x))_{\alpha}$ and $(f(y))_{\beta}$ respectively for which $\mu \not q \nu$. It follows from $m$-continuity of $f$ that $f^{-1}(\mu)$ and $f^{-1}(\nu)$ are fuzzy minimal neighborhoods of $x_{\alpha}$ and $y_{\beta}$ respectively. Since $\mu / q \nu$, so $f^{-1}(\mu) / q f^{-1}(\nu)$. In case that $x=y$ and $\alpha<\beta$ (say), $(f(x))_{\alpha}$ and $(f(y))_{\beta}$ are fuzzy points in $Y$ with $f(x)=f(y)$. Since $(Y, \mathcal{N})$ is fuzzy $m-T_{2}$ space, so $(f(x))_{\alpha}$ has a fuzzy minimal neighborhood $\mu$ and $(f(y))_{\beta}$ has a fuzzy minimal $q$-neighborhoods $\nu$ for which $\mu / q \nu$. Then $f^{-1}(\mu)$ is a fuzzy minimal $q$-neighborhood of $x_{\alpha}$ and $f^{-1}(\nu)$ is a fuzzy minimal $q$-neighborhood of $y_{\beta}$ with $f^{-1}(\mu) \not q f^{-1}(\nu)$. Therefore, $(X, \mathcal{M})$ is fuzzy $m-T_{2}$ space.

Corollary 2.10. Suppose $(X, \mathcal{M})$ and $(Y, \mathcal{N})$ are fuzzy minimal spaces and $f$ : $X \longrightarrow Y$ is injective and fuzzy $m$-continuous. $(X, \mathcal{M})$ is fuzzy $m-T_{i}$ space if $(Y, \mathcal{N})$ is fuzzy $m-T_{i}$ space. 
Proof. It is an immediate consequence of Theorem 2.9 .

Theorem 2.11. Let $(X, \mathcal{M})$ be a fuzzy minimal space. If $(X, \mathcal{M})$ is fuzzy minimal $T_{2}$, then for any two distinct fuzzy points $x_{\alpha}$ and $y_{\beta}$, the following properties hold:

(1) If $x \neq y$, then there exist fuzzy open neighborhoods $\mu$ and $\nu$ of $x_{\alpha}$ and $y_{\beta}$, respectively, such that $m-C l(\nu) \leq \mu^{c}$ and $m-C l(\mu) \leq \nu^{c}$,

(2) If $x=y$ and $\alpha<\beta$ (say), then there exists a fuzzy open neighborhood $\mu$ of $x_{\alpha}$ such that $y_{\beta} \notin m-C l(\mu)$.

Proof. (1) : Let $x \neq y$. Then there exist fuzzy $m$-open neighborhoods $\mu$ and $\nu$ of $x_{\alpha}$ and $y_{\beta}$, respectively, such that $\mu \not q \nu$. Since $\mu \not q \nu$, then $\mu(z) \leq 1-\nu(z)$ and $\nu(z) \leq 1-\mu(z)$ for all $z \in X$. Since $\mu^{c}$ and $\nu^{c}$ are fuzzy $m$-closed, then $m-C l(\nu) \leq \mu^{c}$ and $m-C l(\mu) \leq \nu^{c}$.

(2) : Let $x=y$. Then there exist a fuzzy minimal $q$-neighborhood $\lambda$ of $y_{\beta}$ and a fuzzy open neighborhood $\mu$ of $x_{\alpha}$ such that $\lambda \not q \mu$. Now, let $\nu$ be a fuzzy $m$-open set in $X$ such that $y_{\beta} q \nu$ and $\nu \leq \lambda$. Since $\beta>1-\nu(y)=\left(m-C l\left(\nu^{c}\right)\right)(y), \nu \leq \lambda$ and $\mu \leq \lambda^{c}$, then $\beta>m-C l(\mu)(y)$ for all $y \in X$. Thus, $y_{\beta} \notin m-C l(\mu)$.

Theorem 2.12. Let $(X, \mathcal{M})$ be a fuzzy minimal space. Suppose that $(X, \mathcal{M})$ enjoys the property $U$. Then $(X, \mathcal{M})$ is fuzzy minimal $T_{2}$ if and only if for any two distinct fuzzy points $x_{\alpha}$ and $y_{\beta}$, the following properties hold:

(1) If $x \neq y$, then there exist fuzzy $m$-open neighborhoods $\mu$ and $\nu$ of $x_{\alpha}$ and $y_{\beta}$, respectively, such that $m-C l(\nu) \leq \mu^{c}$ and $m-C l(\mu) \leq \nu^{c}$,

(2) If $x=y$ and $\alpha<\beta$ (say), then there exists a fuzzy $m$-open neighborhood $\mu$ of $x_{\alpha}$ such that $y_{\beta} \notin m-C l(\mu)$.

Proof. $(\Rightarrow)$ : It follows from Theorem 2.11.

$(\Leftarrow)$ : Let $x_{\alpha}$ and $y_{\beta}$ be distinct fuzzy points in $X$ and let $x \neq y$. Then there exist fuzzy $m$-open neighborhoods $\mu$ and $\nu$ of $x_{\alpha}$ and $y_{\beta}$, respectively, such that $m-C l(\nu) \leq \mu^{c}$. This implies that for all $z \in X, \mu(z)+\nu(z) \leq(m-C l(\nu))(z)+$ $\mu(z) \leq 1$. Hence, $\mu / q \nu$. Now, let $x=y$ and $\alpha<\beta$. Then there exists a fuzzy $m$-open neighborhood $\mu$ of $x_{\alpha}$ such that $y_{\beta} \notin m-C l(\mu)$. Let $\lambda=(m-C l(\mu))^{c}$. Since for all $z \in X, \lambda(z)+\mu(z) \leq 1$, then $\lambda / q \mu$. On the other hand, $\lambda$ is a fuzzy open set and $\beta+\lambda(y)>\alpha+\lambda(y) \geq 1$. Hence, $\lambda$ is a fuzzy minimal q-neighborhood of $y_{\beta}$ such that $\lambda \not q \mu$.

Theorem 2.13. Let $(X, \mathcal{M})$ be a fuzzy minimal space. If $(X, \mathcal{M})$ is fuzzy minimal $T_{2}$, then the following hold:

(1) for every fuzzy point $x_{\alpha}$ in $X, x_{\alpha}=\bigwedge\{m-C l(\nu): \nu$ is a fuzzy minimal neighborhood of $\left.x_{\alpha}\right\}$.

(2) for every $x, y \in X$ with $x \neq y$, there exist a fuzzy minimal neighborhood $\mu$ of $x_{1}$ such that $y \notin \operatorname{supp}(m-C l(\mu))$.

Proof. (1) : Let $y_{\beta} \notin x_{\alpha}$. We shall show the existence of a fuzzy minimal neighborhood of $x_{\alpha}$ such that $y_{\beta} \notin m-C l(\nu)$.

Let $x \neq y$. Then there exist fuzzy minimal open sets $\mu$ and $\nu$ containing $y_{1}$ and $x_{\alpha}$, respectively such that $\mu \not q \nu$. Then $\nu$ is fuzzy minimal neighborhood of $x_{\alpha}$ and $\mu$ is a fuzzy minimal q-neighborhood of $y_{\beta}$ such that $\mu / q \nu$. Hence, by using Theorem 2.4 we get $y_{\beta} \notin m-C l(\nu)$. 
Let $x=y$. Then $\alpha<\beta$ and there exist a fuzzy minimal q-neighborhood $\mu$ of $y_{\beta}$ and fuzzy minimal neighborhood $\nu$ of $x_{\alpha}$ such that $\mu \not q \nu$. Thus, $y_{\beta} \notin m-C l(\nu)$.

(2) : For every $x, y \in X$ with $x \neq y$, since $(X, \mathcal{M})$ is fuzzy minimal $T_{2}$, then there exist fuzzy minimal open sets $\mu$ and $\nu$ such that $x_{1} \in \mu, y_{1} \in \nu$ and $\mu \not q \nu$. Then $\nu^{c}(y)=0$ and $\mu \leq \nu^{c}$. Since $\nu^{c}$ is fuzzy minimal closed, $m-C l(\mu) \leq \nu^{c}$. Thus, $m-C l(\mu)(y)=0$ and hence, $y \notin \operatorname{supp}(m-C l(\mu))$.

Theorem 2.14. Let $(X, \mathcal{M})$ be a fuzzy minimal space with property $U$. Then $(X, \mathcal{M})$ is fuzzy minimal $T_{2}$ if and only if

(1) for every fuzzy point $x_{\alpha}$ in $X, x_{\alpha}=\bigwedge\{m-C l(\nu)$ : $\nu$ is a fuzzy minimal neighborhood of $\left.x_{\alpha}\right\}$.

(2) for every $x, y \in X$ with $x \neq y$, there exist a fuzzy minimal neighborhood $\mu$ of $x_{1}$ such that $y \notin \operatorname{supp}(m-C l(\mu))$.

Proof. $(\Rightarrow)$ : It follows from Theorem 2.13 .

$(\Leftarrow)$ : Let $x_{\alpha}$ and $y_{\beta}$ be two distinct fuzzy points in $X$.

Let $x \neq y$. Suppose that $0<\alpha<1$. There exists a real number $\delta$ such that $0<\alpha+\delta<1$. By hypothesis, there exists a fuzzy minimal neighborhood $\mu$ of $y_{\beta}$ such that $x_{\delta} \notin m-C l(\mu)$. Then $x_{\delta}$ has a fuzzy minimal q-neighborhood $\nu$ such that $\mu \not q \nu$. On the other hand, $\delta+\nu(x)>1$ and $\nu(x)>1-\delta>\alpha$ and hence $\nu$ is a fuzzy minimal neighborhood of $x_{\alpha}$ such that $\mu \not q \nu$, where $\mu$ is a fuzzy minimal neighborhood of $y_{\beta}$. If $\alpha=\beta=1$, by hypothesis there exists a fuzzy minimal neighborhood $\mu$ of $x_{1}$ such that $m-C l(\mu)(y)=0$. Thus, $\nu=(m-C l(\mu))^{c}$ is a fuzzy minimal neighborhood of $y_{1}$ such that $\mu \not q \nu$.

Let $x=y$ and $\alpha<\beta$. Then there exists a fuzzy minimal neighborhood of $x_{\alpha}$ such that $y_{\beta} \notin m-C l(\mu)$. Thus, there exists a fuzzy minimal q-neighborhood $\nu$ of $y_{\beta}$ such that $\mu \not q \nu$. Hence, $(X, \mathcal{M})$ is fuzzy minimal $T_{2}$.

\section{ACKNOWLEDGEMENTS:}

This paper is supported by the Research Center in Algebraic Hyperstructures and Fuzzy Mathematics, University of Mazandaran, Babolsar, Iran.

\section{REFERENCES}

[1] M. Alimohammady, S. Jafari and M. Roohi, Fuzzy minimal connected sets, Bull. Kerala Math. Assoc., 5 (2008), 1-15.

[2] M. Alimohammady and M. Roohi, Compactness in fuzzy minimal spaces, Chaos, Solitons \& Fractals, 28 (2006), 906-912.

[3] M. Alimohammady and M. Roohi, Fuzzy minimal structure and fuzzy minimal vector spaces, Chaos, Solitons \& Fractals, 27 (2006), 599-605.

[4] M. Alimohammady and M. Roohi, Fuzzy transfer minimal closed multifunctions, Ital. J. Pure Appl. Math., 22 (2007), 67-74.

[5] M. Alimohammady and M. Roohi, Fuzzy $U_{m}$ sets and fuzzy $(U, m)$-continuous functions, Chaos, Solitons, \& Fractals 28 (2006), 20-25.

[6] M. Alimohammady and M. Roohi, $\Lambda_{m}$-Set in fuzzy minimal space, Journal of Basic Science, 3 (2006), 11-17.

[7] M. Alimohammady and M. Roohi, Separation of fuzzy sets in fuzzy minimal spaces, Chaos, Solitons \& Fractals, 31 (2007), 155-161. 
[8] A. Azam, M. Arshad and I. Beg, Common fixed point theorems in cone metric spaces, J. Nonlinear Sci. Appl., 2 (2009), 204-213.

[9] B. Ghosh, Semi-continuous and semi-closed mappings and semi-connectedness in fuzzy setting, Fuzzy sets syst. 35 (1990), 345-355.

[10] I. M. Hanafy, $\beta S^{*}$-compactness in $L$-fuzzy topological spaces, J. Nonlinear Sci. Appl., 2 (2009), 27-37.

[11] S. Jafari, K. Viswanathan, M. Rajamani and S. Krishnaprakash, On decomposition of fuzzy A-continuity, J. Nonlinear Sci. Appl., 1 (2008), 236-240.

[12] P. Pao-Ming and L. Ying-Ming, Fuzzy topology I. neighborhood structure of a fuzzy point and and Moore-Smith convergence, J. Math. Anal. Appl. 76 (1980), 571-599.

[13] P. Pao-Ming and L. Ying-Ming, Fuzzy Topology II. Product and quotient spaces, J. Math. Anal. Appl., 77 (1980), 20-37.

[14] L. A. Zadeh, Fuzzy sets, Inf. control, 8 (1965), 338-353.

${ }^{1}$ Department of Mathematics, Faculty of Basic Sciences, University of MazanDARAN, BABOLSAR 47416 - 1468, IRAN

E-mail address: amohsen@umz.ac.ir

E-mail address: mehdi.roohi@gmail.com

2 Department of Mathematics, Canakkale Onsekiz Mart University, Terzioglu Campus, 17020 Canakkale, Turkey

E-mail address: eekici@comu.edu.tr

3 Department of Economics, Copenhagen University, Oester Farimagsgade 5, Building 26, 1353 Copenhagen K, Denmark

E-mail address: jafari@stofanet.dk 